\title{
Active Contour Multigrid Model for Segmentation and Automatic Quantification of Material Phases of Cast Iron
}

\author{
Pattan Prakash \\ Dept. of Computer Science \\ and Engineering, \\ PDA College of Engineering, \\ Gulbarga -585102,India
}

\author{
V. D. Mytri \\ GND College of \\ Engineering \\ Bidar-585403, India
}

\author{
P. S. Hiremath \\ Dept. of Computer Science, \\ Gulbarga University, \\ Gulbarga.-585106,India
}

\begin{abstract}
A critical and important stage in microstructure image analysis is segmentation, because the segmentation method has direct impact on the end results of analysis. The main aim of this paper is to determine appropriate segmentation method for microstructure image analysis and quantification. In this work, some popular segmentation methods, namely, Otsu's automatic threshold, watershed, uni-grid active contour method and multi-grid active contour methods have been investigated. The reliability of the segmentation methods is tested by determining the volume fraction of phases present in microstructure images of materials of known chemical composition. The experimentation is done using microstructure images of cast iron of various compositions. The experimental results are compared with expected values of volume fraction. The active contour multi-grid segmentation model is found to yield better results within the practical limits as compared to manual and other automated methods.
\end{abstract}

Keywords: phase of material, microstructure, metallograph, knowledge-base, volume fraction, watershed, active contour.

\section{INTRODUCTION}

It is well known that there is direct relationship between the microstructure and the properties of the material. Metals are crystalline when they are in the solid form. The crystal structure of a solid metal refers to the internal structure or arrangement of the atoms in an ordered, periodic, 3D pattern. Normal metallic objects are polycrystalline, which means they consist of an aggregate of many minute crystals. These crystals are called grains. Some metallic objects, such as castings, have very large grains that can be resolved with the naked eye and these structures are referred to as macrostructures. Typically, the grains of a metal object are very small, and can not be viewed with the naked eye. The structural features of the small grains are observed using an optical microscope or metallograph, or an electron microscope, at magnifications greater than 100 times. Structures requiring this range of magnification for their examination are called microstructures. Some sample microstructure images are shown in Fig 1.

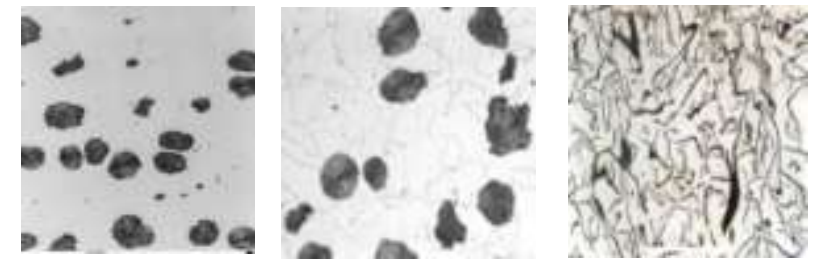

Fig 1.Sample microstructure images of cast iron showing the two phases, pearlite and ferrite.
The macrostructural and microstructural examination techniques are employed in areas such as routine quality control, failure analysis and research studies. In quality control, microstructural analysis is used to determine if the structural parameters are within certain permissible limits. It is used as a criterion for acceptance or rejection. The microstructural features, normally, are grain size, amount of impurities, second phases, porosity, segregation and volume fraction of a phase or defects present.

\subsection{Phase of material and its volume fraction $(\boldsymbol{V} v)$}

In the materials science, the word "phase" usually refers to the particular arrangement of atoms in a material [3]. For example, the atoms in a piece of cast iron can form different arrangements, depending on its temperature and purity. For example, a piece of cast iron that contains a mixture of graphite and ferrite could be called a two-phase material with graphite (pearlite) and ferrite as its two phases.

The volume fraction is an important measure of relative amount of a phase in a material. The phase in a material is governed by the chemistry and the processing history of the material. Many properties of the materials depend on the constitution of different phases in a material. For example, the tendency of delayed cracking of quenched material depends on the relative amount of retained austenite (phase). By knowing the chemical composition of the material, the volume fraction of a phase, like graphite, in cast iron is computed by using the formula (1) [3]:

$V v($ graphite $)=\frac{7.8 * \% C}{222+5.6 * \% C}$

where $\% C$ is carbon content. There are many manual methods, in practice, for quantitative microscopy such as line intersection method, circle intersection method [3] and many more. Digital image processing techniques offer better alternative methods, which minimize human efforts and, also, save a lot of time. For digital measurement, the following relation is used for volume fraction [3]:

$$
V v=\frac{N_{p}}{N_{o}}
$$

where $N_{p}$ is the number of pixels that belong to the phase being analyzed, and $N_{o}$ is the total number of pixels in the image. 


\subsection{Importance of segmentation stage in microstructure image analysis}

In most image analysis operations, pattern classifiers require individual objects to be separated from the background in the image. The description of those objects needs to be transformed into a suitable form for computer processing. Image segmentation is a fundamental task responsible for such operation. The function of segmentation is to partition an image into its constituent and disjoint sub-regions, which are uniform according to their properties, e.g. intensity, color, and texture. Segmentation algorithms are generally based on either discontinuity among subregions, i.e. edges, or uniformity within a sub-region, though there are some segmentation algorithms relying on both discontinuity and uniformity.

The segmentation critically affects the results of classification and quantification in microstructure image analysis. It often determines the eventual success or failure of the image analysis. The level to which segmentation is carried depends on the problem being solved. That is, segmentation should stop when the region of interest (ROI) in the application has been isolated. Due to this property of problem dependence, segmentation is one of the most difficult tasks in image analysis. Noise and mixed pixels caused by the poor resolution of sensor images make the segmentation problem even more difficult and quantification results are often disagreed. In this paper, we examine the performance of four popular segmentation methods, namely, Otsu's optimum threshold [5], watershed [4,14,15], active contours $[8,11,12]$.

\subsection{Background literature}

Many efforts have been made to achieve the optimum results in image segmentation. To mention a few, the threshold based method is proposed by Otsu [5]. Contour-based segmentation methods are also of interest of many authors and, among them, Marr-Hilderth method is discussed in [10]. Edge detection techniques are discussed by Canny in [11]. A series of morphological operations for segmentation of microstructures are effectively used in [6,7]. But these methods are dependent on the selection of appropriate size of structural element. The object identification by using snakes, shapes and gradient vector flow is discussed by Michael Kass [12]. The active contour based method is discussed by Chan and Vese in [8] and an improved active contour multi-grid model is discussed by G. Papandreou and P.Maragos [20]. The minimum distance classifier for phase discrimination is discussed in [13]. Various contour based and region based methods are extensively discussed in $[1,2,4]$. With this background and motivation, it is felt that a robust segmentation method for microstructure analysis is due. The segmentation method should be fully automatic, robust and should provide good segmentation results irrespective of variations in image quality and magnifications.

\subsection{Materials used}

We have used the microstructural images of low-carbon steel (cast iron) captured by using light microscope. The images are of size 200x200 pixels. The samples are taken from steel bars that have graphite and ferrite phases. Images are of various resolutions (i.e. magnifications) and chemical compositions. The etching medium used for preparing specimen is $3 \%$ alcoholic nitric acid.

\section{SEGMENTATION TECHNIQUES}

We consider the segmentation techniques, namely, Otsu's optimum threshold, watershed segmentation and active contour methods (both uni-grid and multi-grid), for investigating the effect of segmentation on classification and quantification of phases of material in a cast iron sample. The motivation for selection of these segmentation methods for investigation is; as these methods are of general choice of many researchers for the segmentation of images in many image processing applications. Their characteristics can vary significantly according to the specific application and imaging modality. Currently, no single existing segmentation scheme will yield acceptable results over the entire spectrum of image types that may be contemplated. A blind selection of a popular segmentation method without a proper study can significantly influence the quantification results. It is imperative to study and propose a suitable segmentation method among the popularly used segmentation methods for the automatic quantification of material phases of cast iron.

\subsection{Otsu's optimum threshold}

We use the Otsu's optimum segmentation method [5] based on thresholding, in which we choose the threshold to minimize the interaction variance of the thresholded black and white pixels. It is based on selecting the lowest point between two peaks of gray level intensity in the histogram of the image. The algorithm assumes the image to be thresholded and containing two classes of pixels (e.g. foreground and background). Then it calculates the optimum threshold separating those two classes so that their combined spread (intra-class variance) is minimal.

\subsection{Watershed segmentation}

The term watershed refers to a ridge that divides areas drained by different river systems. A catchment basin is the geographical area draining into a river or reservoir. In this method, image is treated as topographical surface, low intensity values in the image form the catchment basins and high intensity values form the ridges that separate the adjacent catchment basins $[4,14,15]$.

\subsection{Active contour}

Snakes or active contours, are curves defined within an image domain that can move under the influence of internal forces coming from within the curve itself and external forces computed from the image data. The internal and the external forces are defined so that the snake will confirm to an object boundary or other desired features within an image. Snakes or active contour models they lock onto nearby, edges, localizing them accurately. Scale-space continuation can be used to enlarge the capture region surrounding a feature. The basic idea in active contour models or snakes is to evolve a curve, subject to constraints from a given image $u_{0}$, in order to detect objects in that image. For instance, starting with a curve around the object to be detected, the curve moves toward its interior normal and has to stop on the boundary of the object. Snakes provide a unified account of a number of visual problems, including detection of edges, lines, and subjecting contours; motion tracking; and stereo matching.

Let $\mathrm{C}$ be a planar curve with length $\mathrm{L}(\mathrm{C})$ and $\vec{C}(s) \equiv$ $(x(s), y(s)):[0, L(C)] \rightarrow R^{2}$ its arc-length parameterization; also let I be an intensity image. In geodesic active contours, curve's geodesic length will be minimized [19] 
$\mathrm{EC}=\int_{C} g(|\nabla I(\vec{C}(S))| d s$

where the edge indication function $g:[0,+\infty) \rightarrow R^{+}$is a decreasing function of $|\nabla I|$ (other measures of edge strength can also be used), such that $g(0)=1, g(r) \rightarrow 0$ as $r \rightarrow+\infty$. Minimization of the functional in Eq. (3) by means of variational techniques leads to an Euler-Lagrange partial differential equation (PDA); to reach local minima, we start with an initial contour and evolve it in the direction of steepest decent, introducing a pseudotime variable ' $t$ '. Numerical implementation of the geodesic active contour model using level sets [18] naturally allows for changes in the contour's topology, permitting splits and merges. In the level set framework, the moving contour $\mathrm{C}(\mathrm{t})$ is defined implicitly as the zero level set of an embedding scalar function $\mathrm{u}$ with domain the whole image plane, i.e. $\mathrm{C}(\mathrm{t})=\{(\mathrm{x}, \mathrm{y}): \mathrm{u}(\mathrm{x}, \mathrm{y}, \mathrm{t})=0\}$, where $\mathrm{u}(\mathrm{x}, \mathrm{y}, \mathrm{t}): \mathrm{R}^{2} \mathrm{x}[0,+\infty) \rightarrow R$. By convention, assign negative values to the interior and positive values to the exterior of the courve. The signed distance function from the contour is often chosen for extending $u$ away from the curve due to its good numerical properties.

The corresponding geometric active contour level set evaluation law is

$$
\begin{gathered}
\frac{\partial u}{\partial t}=|\nabla u|\left\{\operatorname{div}\left(\frac{\nabla u}{|\nabla u|}\right)+\alpha / 2\left(\left(f_{R}-I\right)^{2}-\left(f_{\hat{R}}-I\right)^{2}\right)+\right. \\
\left.\beta / 2\left(\left|\nabla f_{R}\right|^{2}-\left|\nabla f_{\dot{R}}\right|^{2}\right)\right\}
\end{gathered}
$$

where, $\alpha$ and $\beta$ are positive weights. Since $f$ is also unknown, it needs to be re-estimated as the front moves. In the Chan-Vese model, where the image intensity is assumed piecewise constant, the curve evolves by

$\frac{\partial u}{\partial t}=|\nabla u|\left\{\operatorname{div}\left(\frac{\nabla u}{|\nabla u|}\right)+\alpha / 2\left(\left(C_{R}-I\right)^{2}-\left(C_{\hat{R}}-I\right)^{2}\right)\right\}(5)$

Multigrid numerical methods employ a hierarchy of grids of different mesh sizes to efficiency solve a wide range of problems, most notably those arising from PDE models [17]. Multi-grid techniques overcome the typically slow convergence properties of conventional uni-grid iterative methods. In this work, we have employed and compared both uni-grid and multi-grid techniques.

\section{FEATURE EXTRACTION}

The features of the regions of interest (ROIs), namely, graphite and ferrite, are the mean and standard deviation of intensity values of pixels belonging to these regions. Initially, a knowledge base of feature values of pearlite and ferrite phases in microstructure images of known chemical composition of low-carbon steel is built. The knowledge base is given in the Table 1 [16].

Table 1. Knowledge-base of feature values for graphite and ferrite phases in microstructure images of cast iron.

\begin{tabular}{|l|l|l|l|l|l|l|}
\hline Phase & \multicolumn{2}{|l|}{ Otsu } & \multicolumn{2}{l|}{ Watershed } & \multicolumn{2}{|l|}{ Active contour } \\
\cline { 2 - 6 } & $\begin{array}{l}\text { Mean } \\
(\mathrm{M})\end{array}$ & $\begin{array}{l}\text { Std. } \\
\text { Dev. }\end{array}$ & $\begin{array}{l}\text { Mean } \\
(\mathrm{M})\end{array}$ & $\begin{array}{l}\text { Std. } \\
\text { Dev. } \\
(\mathrm{SD})\end{array}$ & $\begin{array}{l}\text { Mean } \\
(\mathrm{M})\end{array}$ & $\begin{array}{l}\text { Std. } \\
\text { Dev. } \\
(\mathrm{SD})\end{array}$ \\
\hline
\end{tabular}

\begin{tabular}{|l|l|l|l|l|l|l|}
\hline & & $(\mathrm{SD})$ & & & & \\
\hline $\begin{array}{l}\text { Grap- } \\
\text { hite }\end{array}$ & $<170$ & 17 & $<190$ & 21 & $<166$ & 19 \\
\hline Ferrite & $>220$ & 10 & $>210$ & 16 & $>210$ & 14 \\
\hline
\end{tabular}

The Table 1 is used to identify graphite and ferrite phases present in the test images. The algorithm for classification and quantification of phases of material is given below. The feature vectors $\mathrm{f}($ library $)=(\mathrm{M}, \mathrm{SD})$ for graphite and ferrite are stored in the feature library (knowledge-base).

\section{Algorithm 1: Training}

Step 1. Input the grayscale microstructure image (training image).

Step 2. Apply preprocessing methods, namely, circular averaging filter with radius 0.5 (empirical value), dilation of filtered image.

Step 3. Apply the segmentation method on pre-processed image of Step 2 and obtain binary image. Perform segment labeling of the binary image.

Step 4. Compute the statistical features, namely, the mean (M) and standard deviation(SD) of intensity values of pixels in labeled segments known to be graphite region or ferrite region. Form feature vectors $\mathrm{f}_{1}=(\mathrm{M}, \mathrm{SD})$ and $\mathrm{f}_{2}=(\mathrm{M}, \mathrm{SD})$ for each graphite and ferrite segment, respectively.

Step 5. Repeat Steps 1 to 4 for all the training images and compute the average of all the feature vectors $f_{1}$ and $f_{2}$ of all the images, and store them as $\mathrm{f}_{1 \text { (library) }}$ and $\mathrm{f}_{2 \text { (library) }}$ in the knowledgebase.

\section{Algorithm 2: Testing}

Step 1: Input the grayscale microstructure image (test image).

Step 2: Apply preprocessing methods, namely, circular averaging filter with radius 0.5 (empirical value), dilation of filtered image.

Step 3: Apply the segmentation method on pre-processed image of Step 2 and obtain binary image. Perform segment labeling of the binary image.

Step 4: For each labeled segment, compute the statistical features, namely, the mean (M) and standard deviation (SD) of intensity values of pixels and form feature vector $\mathrm{f}($ test $)=(\mathrm{M}, \mathrm{SD})$.

Step 5: Compute the Euclidean distance $D$ between the feature vector $\mathrm{f}$ (test) of each segment of test image and feature vectors $\mathrm{f}$ (library) stored in the feature library.

$$
D=\sqrt{\sum_{i=1}^{n}\left(f_{(\text {test }) i}-f_{(\text {library }) i}\right)^{2}} .
$$

Step 6: Apply minimum distance classifier to classify the labeled segments as graphite and ferrite phases. Compute the total area occupied by each phase in the image.

Step 7: Compute volume fraction $V v$ for both phases in the input image using the equation (2).

Step 8 : Repeat Steps 1 to 7 for all the test images and compute the average volume fraction $V v$ of each phase for the sample material, which is the estimation of the volume fraction of the phases. 
The above algorithms are implemented by using four different segmentation methods, namely, Otsu's optimum threshold method, watershed method, uni-grid active contour method and multi-grid active contour method, in the Step 3. However, in case of active contours (both, in uni-grid and multi-grid), the preprocessing operations of Step 2 are not employed. The performance of four segmentation methods in terms of accuracy of volume fraction is compared.

\section{EXPERIMENTAL RESULTS}

For experimentation, we have used 100 microstructural images of low-carbon steel samples taken from steel bars, with graphite and ferrite phases, captured using light microscope. Images are of size $200 \times 200$ pixels. The etching medium used for preparing specimen is $3 \%$ alcoholic nitric acid. These images are obtained from the microstructure library [9]. We have used 75 microstructure images for building knowledge-base and remaining are used for testing. We have employed Otsu's optimum thresholding, watershed and active contour methods (uni-grid and multi-grid) of segmentation on the same set of images and computed the volume fraction using the equation (2). In the case of active contour multi-grid model, the multi-grid model is initiated by multi-grid circular masks (as shown in Fig. 2) on whole image to obtain segmented regions in the image.

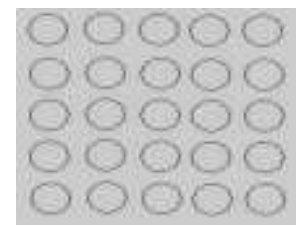

Fig. 2 Multi-grid circular mask

The segmentation results are shown in the Fig. 3 and the computed volume fraction of the graphite and ferrite phases are shown in the Table 2 .

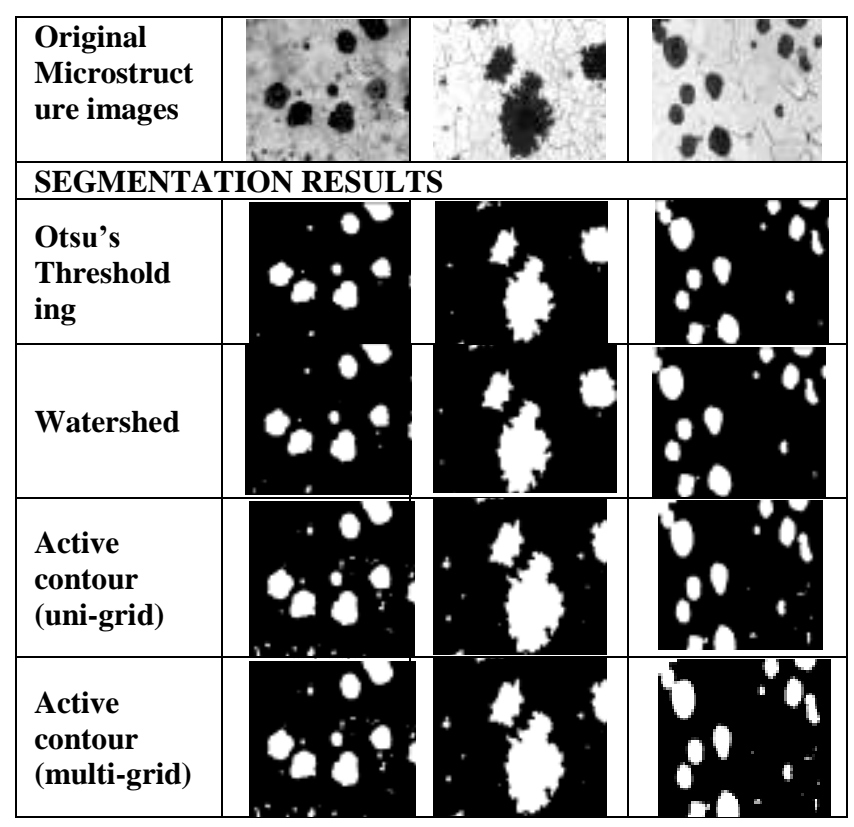

Table 2. Comparison of volume fraction $(V v)$ of phases obtained by using proposed method and expected volume fraction $(V v e)$ for different segmentation methods.

\begin{tabular}{|c|c|c|c|c|c|c|c|}
\hline Image & $\begin{array}{l}\text { Vve } \\
\text { (Grap } \\
\text { hite) } \\
(\%)\end{array}$ & $\begin{array}{l}\text { Vve } \\
\text { (Ferri } \\
\text { te) } \\
(\%)\end{array}$ & $\begin{array}{l}\text { Segmenta } \\
\text { tion } \\
\text { Method }\end{array}$ & $\begin{array}{l}\boldsymbol{V} \boldsymbol{v} \\
\text { (Graphi } \\
\text { te) } \\
(\%)\end{array}$ & $\begin{array}{l}\text { Difference } \\
\boldsymbol{V} \boldsymbol{v e} \sim \boldsymbol{V} \boldsymbol{v} \\
\text { (Graphite) }\end{array}$ & $\begin{array}{l}\boldsymbol{V v} \\
\text { (Ferri } \\
\text { te) } \\
(\%)\end{array}$ & $\begin{array}{l}\text { Differenc } \\
\text { e } \\
\underset{\text { (Ferrite) }}{\boldsymbol{v} e \sim \boldsymbol{V}}\end{array}$ \\
\hline \multirow{4}{*}{$\begin{array}{l}00006 \\
4[1]\end{array}$} & \multirow{4}{*}{11.59} & \multirow{4}{*}{81.0} & $\begin{array}{l}\text { Threshold } \\
\text { ing [16] }\end{array}$ & 14.32 & 2.73 & 79 & 4 \\
\hline & & & $\begin{array}{l}\text { Watershe } \\
\mathrm{d}[16]\end{array}$ & 15.50 & 3.91 & 88 & 7 \\
\hline & & & 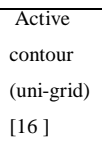 & 12.50 & 0.91 & 82 & 1 \\
\hline & & & $\begin{array}{l}\text { Active } \\
\text { contour } \\
\text { (multi- } \\
\text { grid) }\end{array}$ & 11 & 0.59 & 80.5 & 0.5 \\
\hline \multirow{4}{*}{$\begin{array}{l}00006 \\
4[1] 2\end{array}$} & \multirow{4}{*}{11.59} & \multirow{4}{*}{80.0} & $\begin{array}{l}\text { Threshold } \\
\text { ing [16] }\end{array}$ & 14.32 & 2.73 & 85 & 5 \\
\hline & & & $\begin{array}{l}\text { Watershe } \\
\mathrm{d}[16]\end{array}$ & 15.50 & 3.91 & 88 & 8 \\
\hline & & & $\begin{array}{l}\text { Active } \\
\text { contour(u } \\
\text { ni-grid) } \\
{[16]}\end{array}$ & 12.50 & 0.91 & 76 & 4 \\
\hline & & & $\begin{array}{l}\text { Active } \\
\text { contour } \\
\text { (multi- } \\
\text { grid) }\end{array}$ & 12.00 & 0.41 & 77 & 3 \\
\hline
\end{tabular}

The rate of classification of phases is $100 \%$ and the quantification results are very close to the corresponding expected values. The average quantification error is $0.8 \%$ for graphite and $2.3 \%$ for ferrite. The comparison of error in computed volume fraction values with expected values for both phases obtained by different segmentation methods is shown in Fig. 4 and Fig. 5.

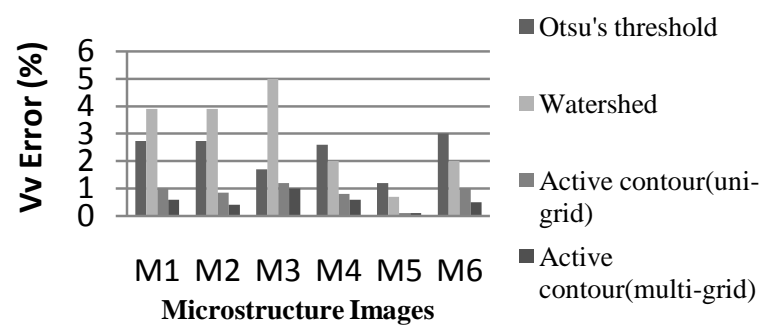

Fig 4. Comparison of error in computed volume fraction values with expected values for graphite obtained by three segmentation methods.

Fig 3. Segmentation results of four methods 


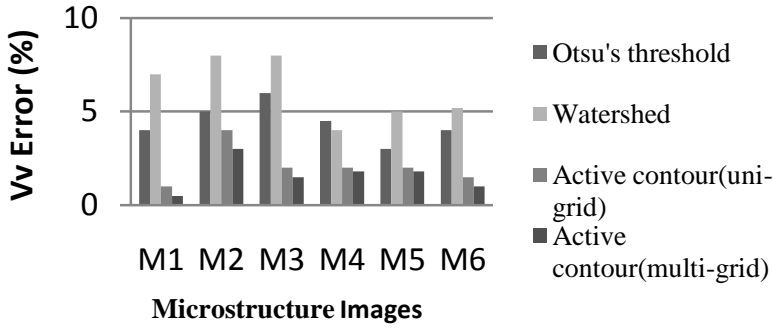

Fig 5. Comparison of error in computed volume fraction values with expected values for ferrite obtained by three segmentation methods.

It is observed that the estimation of volume fraction $(V v)$ of phases in microstructure images using multi-grid active contours segmentation method is more accurate, as compared to other methods, despite heavy noise in the input microstructure images. In Otsu's optimum thresholding and watershed segmentation methods, the pre-processing plays a vital role in achieving good segmentation results. In the case of microstructures having fine grain structures, it is observed that over estimation of volume fraction is due to over segmentation owing to heavy noise content. But multi-grid active contours segmentation method performed well even in the case of heavy noise present in microstructure images. Multi-grid techniques overcome the typically slow convergence properties of conventional uni-grid iterative methods. While uni-grid relaxation procedures are particularly effective at eliminating the high spatial frequency part of error, they suppress very slowly its low-frequency part. This behavior stems from the local nature of computations in uni-grid methods, which allows attenuation of error components that vary on scales comparable to the discretization mesh grid size $h$, but prevents quick suppression of larger scale errors. As discretization grids gets finer, information propagates even more slowly and the local effects of uni-grid methods become more pronounced. Multi-grid cycles overcome these difficulties by employing conventional relaxation procedures, called as smootheners in multi-grid terminology, in a hierarchy of grids. Since relaxation performed at each resolution level smoothens the error component at scales comparable to the grid size of this level, one multi-grid can effectively eliminate error component at the whole range of frequencies. Moreover, applying a relaxation procedure at coarse scales cause significantly less than applying it at fine scales, since less variables are involved, which makes active contour method as more robust method for automatic classification and quantification of phases of material.

\section{CONCLUSIONS}

In this paper, we investigate the performance of segmentation methods for automatic quantification of material phases of cast iron, in order to determine appropriate segmentation method for microstructure image analysis and quantification. Some popular segmentation methods, namely, Otsu's automatic threshold, watershed and active contour methods, both uni-grid and multigrid, have been investigated. The reliability of the segmentation methods is tested by determining the volume fraction of phases present in microstructure images of materials of known chemical composition. The experimentation is done using microstructure images of cast iron of various compositions. The experimental results are compared with expected values of volume fraction. The multi-grid active contour segmentation method is found to yield better results within the practical limits as compared to other methods, which makes active contour method as more robust method for automatic classification and quantification of phases of material. Although, the quantification results obtained using unigrid and multi-grid active contour segmentation methods are very close, but it is observed that the uni-grid active contour segmentation method requires more segmentation time.

\section{ACKNOWLEDGEMENTS}

The authors are grateful to the referees for their valuable comments and suggestions. Further, the authors are indebted to Dr. G.K. Purohit, Prof. Ravindra M.Latthe and Prof. Basawanand Motgi, Mechanical Engineering Department of PDA College of Engineering, Gulbarga, India for providing domain knowledge and manual characterization of microstructure images.

\section{REFERENCES}

[1] A.K.Jain, Fundamentals of Digital Image Processing. PrenticeHall, Englewood Cliffs, NJ,1989

[2] R.C. Gonzalez and R.E. Woods, Digital Image Processing, Addison-Wesley,1993.

[3] Handbook Committee, Hand book of ASM International, Vol 9, Metallography and Microstructures, ISBN:0-87170-706-3.

[4] Milan Sonka, Vaclav Hlavac, Roger Boyle, Image Processing, Analysis, and Machine Vision, 2e. PWS Publishing, ISBN:81-315-0300-3, ISSN:978-81-315-0300-3, 1999.

[5] Otsu N., A Threshold Selection Method from Grayscale Histograms, IEEE Transaction Systems Man Cybernetics SMc-9, 1979,pp. 62-66.

[6] Pattan Prakash, V.D.Mytri, P.S.Hiremath, Classification of Cast Iron Based on Graphite Grain Morphology using Neural Network Approach, International Journal of Engineering and Technology(IJENGG), Volume 2, Number 4, December 2009, pp-38-42.

[7] Pattan Prakash, V.D.Mytri, P.S.Hiremath, Classification of Cast Iron Based on Graphite Grain Morphology using Neural Network Approach, Proc. of SPIE Vol 546, Second Inter National conference on Digital Image Processing 2010 (ICDIP 2010), Singapore, Feb 26-28, 2010, pp. 75462S-1 75462S-6.

[8] T.F. Chan and L. Vese, IEEE Trans. on Image Processing, Vol. 10, No. 2, Feb 2001, pp. 266-277.

[9] Microstructure Libraries: http://www.metalograf.de/starteng.htm, www.doitpoms.ac.uk

[10] D. Marr and E.Hilderth, On the Theory of Edge Detection, Proc. R.Soc.(London) B, Vol 207, Royal Society of London, 1980, pp. 127-217.

[11] J.Canny, A computational Approach to Edge Detection, IEEE Transaction, Pattern Nov 1986, pp. 679-698.

[12] Michael Kass, Andrew Witkin and Demitri, Snakes:Active contour models, International Journal of Computer Vision, 1998, pp. 321-331. 
[13] Pattan Prakash, V.D. Mytri and P.S. Hiremath, Automatic Microstructure Image Analysis For Classification And Quantification of Phases of Material, International Conference on Systemics, Cybernetics and Informatics (ICSCI 2009), Jan7-10,2010, pp. 330-333.

[14] Dobrin B.P., Viero T and Gabbouj M., Fast Watershed Algorithms: Analysis and Extensions, Proceedings of SPIE, Vol. 2180, 1994, pp. 209-220.

[15] Meyer, Fernand, "Topographic distance and watershed lines", Signal Processing, Vol. 38, July 1994, pp. 113-125.

[16] Pattan Prakash, V.D. Mytri and P.S.Hiremath, Performance Analysis of Segmentation Methods for Automatic Quantification of Phases of Cast Iron, Proc. of Int'l Conf. on
Computational Vision and Robotics (ICCVR)- 2010, Aug 21-22, 2010, pp. 174-180.

[17] A. Brandt, Multi-level Adaptive Solutions to Boundary-value Problems, Math. Comp. Vol. 31 No. 138, 1977, pp.333-390.

[18] S.Oshar and J.A.Sethian, Fronts Propagating with Curvature Dependent Speed: Algorithm Based on Hamilton Jacobi Formulation, J.Compact Phy. Vol.79, 1988, pp. 12-49.

[19] V. Caselles, R.Kimmel and G. Sapiro, Geodesic Active Contours, Int'1 J. Comp. Vision, Vol 22 No.1, 1977, pp.6169.

[20] George Papandreou, Petros Magagos, Multigrid Geometric Active Contour Models, IEEE Trans. on Image Proc. Vol. 16, 2007, pp. 229-240. 